\title{
Efficacy of acarbose in different geographical regions of the world: analysis of a real-life database
}

SANJAY KALRA ${ }^{1}$, SIDARTAWAN SOEGONDO², OLIVER SCHNELL ${ }^{3}$, WAYNE H SHEU ${ }^{4}$, WLADYSLAW GRZESZCZAK ${ }^{5}$, HIROTAKA WATADA ${ }^{6}$, NORIYUKI YAMAMOTO ${ }^{7}$, JIANPING WENG ${ }^{8}$ ${ }^{1}$ Bharti Research Institute of Diabetes \& Endocrinology, Haryana, India; ${ }^{2}$ University of Indonesia, Jakarta, Indonesia; ${ }^{3}$ Forschergruppe Diabetes e.V., Helmholtz Center, Munich, Germany; Division of Endocrinology and Metabolism, Department of Internal Medicine, Taichung Veterans General Hospital, Taichung, Taiwan; ${ }^{5}$ Silesian University of Medicine, Zabrze, Poland; Juntendo University Graduate School of Medicine, Tokyo, Japan; 'Bayer Healthcare, Beijing, China; ${ }^{8}$ Third Affiliated Hospital of Sun Yat-Sen University, Guangzhou, China.

\section{Abstract}

Introduction: Although alpha-glucosidase inhibitors (aGls), including acarbose worldwide due to a perception that they are less effective in Caucasians than Asians. A study was undertaken to
ethnicity/region populations exist.

Methods/design: We pooled data from 10 non-interventional and post-marketing acarbose to 2011. The effects of acarbose on glycosylated hemoglobin ( $\mathrm{Hb}$ A $A_{10}$ ) fasting plasma glucose (FPG) and post-prandial plasma glucose (PPG) were from East, South East and South Asia.

Results: The efficacy population included 62,905 patients, with 59,090 patients

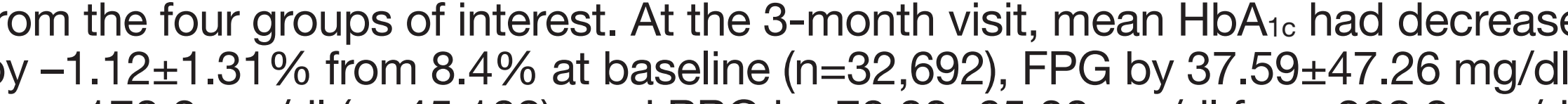
from $170.2 \mathrm{mg} / \mathrm{dl}(\mathrm{n}=45,102)$, and PPG by $70.00 \pm 65.30 \mathrm{mg} / \mathrm{dl}$ from $238.2 \mathrm{mg} / \mathrm{dl}$ larger in patients with higher baseline values regardless of ethnicity and region. Data from 30,730 patients from the four groups with non-missing baseline and 3-month
HbAtc data, age and sex were analyzed by multivariable ANCOVA. After adjustmen better responses to acarbose than South Asians and European Caucasians,
owever, the differences were numerically small (e.g. relative difference of $\sim 2.2 \% \mathrm{for}$ baseline $\mathrm{HbA}_{1 \mathrm{c}}$ of $7.2 \% ; \sim 3.4 \%$ for baseline $\mathrm{HbA}_{1 \mathrm{c}}$ of $9.2 \%$ ). In the safety population $(\mathrm{n}=67,682)$, acarbose was well tolerated, with few ep
$(0.03 \%)$ and gastrointestinal adverse events $(2.76 \%)$

Conclusion: Acarbose was effective in European Caucasians and Asians; however slightly better responses to acarbose than South Asians and European Caucasians. Introduction

Acarbose, the most widely prescribed of the currently available alpha-glucosidase
inhibitors (aGls), has been used in the management of hyperglycemia for more than

- Acarbose acts non-systemically to slow down carbohydrate digestion and
attenuates high levels of post-prandial plasma glucose (PPG), which is:-2:9

an important manifestation in the early course of type 2 diabetes mellitus

(T2DM)

a contribal target for achieving factor in the pathogenesis of cardiovascular disease

Acarbose indirectly optimizes glucose metabolism during the day through
adaptatito of insulin secretion?

Numerous studies have shown beneficial effects of acarbose as a $1 \mathrm{st}-$, 2nd- and
3rd-line treatment option, $10-28$ for which it is recommended by both the Internationa

Diabetes Federation (IDF) and American Association for Clinical Endocrinologists

However, prescribing of acarbose varies around the work
efficacy is limited in some ethnic and regional groups

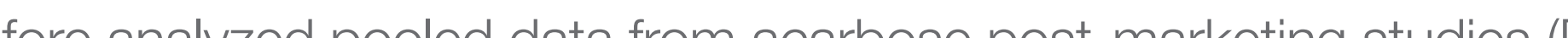

non-interventional studies (NIS) to examine whether differences between patients of Methods

Data from 10 PMS and NIS from 21 countries, provinces and country groups acros
the world from the launch of acarbose to 2001 were pooled in a single database Data on PPG, fasting blood glucose (FBG), glycosylated hemoglobin ( $\left(\mathrm{Hb} \mathrm{A}_{1 \mathrm{t}}\right.$ ), and/or body weight collected
efficacy of acarbose

Bivariate analysis of covariance (ANCOVA) models were used to assess the relative reduction in $\mathrm{Hb} \mathrm{A}_{1 \mathrm{c}}$ versus baseline $\mathrm{Hb} \mathrm{A}_{1 \mathrm{c}}$, with adjustment for base
subgroup of interest and baseline $\mathrm{Hb} \mathrm{A}_{1 \mathrm{c}}$ by subgroup interaction

Multivariable ANCOVA was used to compare HbA $A_{1}$ response to acarbose in
terms of relative change in $H$ b $A_{1}$ at 3 months versus baseline, with adjustment for baseline HbA 1 c, ethnicity/region and ethnicity/region group interactions (baseline value, ethnicity/region subgroup, pretreatment, disease duration, baseline HbAtc by
subgroup, disease duration by subgroup, dose category, body mass index (BMI)

Results

Patient population

(67,682 patents were included in the satety population

After excluding patients who were retrospectively documented, did not take
acarbose, did not have follow-up visits, were younger than 18 years and did not have at least two measurements of FPG and PPG, the efficacy population comprised data

(1) East Asian Asians from China, Hong Kong, Taiwan, Japan and South Korea European Caucasians from Bosnia and Herzegovina, Germany, Poland and South Asian Asians from India and Pakistan (n=8,738: 15\%)

South East Asian Asians from Cambodia, Indonesia, Malaysia, Philippines,

After restriction of the original efficacy population to the four major ethnicity/region groups and additional exclusions, including patients with missing baseline and/or
post-treatment $H b A_{1}$ data, 30,730 subjects were considered for the multivariable Mean follow up ( \pm SD) for the efficacy population with non-missing post-treatment FPG or PPG value(s) was 12.2 4.8 (range 0.1-108.9) weeks

Patient characteristics

Gender percentages were balanced, except for a higher percentage of men in the

Elderly patients were more common in the European and East Asian groups (median
age 63 and 61 years, respectively) than South East Asian and South Asian groups median age 55 and 49 years, respectively)

Patients were heavier in Europe and South Asia (median weight 84 and $74 \mathrm{~kg}$,
respectively) than in East Asia and South East Asia (median weight 67 and $65 \mathrm{~kg}$,

The proportion of obese patients (using the Western standard definitions for

European Caucasians and the Asia Pacific standard for Asians) was higher for South

Hypertension and dyslipidemia were more common in European patients $(71.6 \%$ and Cerebrovascular accident/stroke was more common in East Asian (11.0\%) and
European (8.2\%) patients than South East Asian (2.4\%) and South Asian (1.8\%) Microvascular comorbidities did not differ greatly between ethnicity/region groups Table 1 summarizes the baseline values for $\mathrm{HbA}_{1,}, \mathrm{FPG}$ and PPG for the efficacy
population overall and the four ethnicity/region groups under evaluation

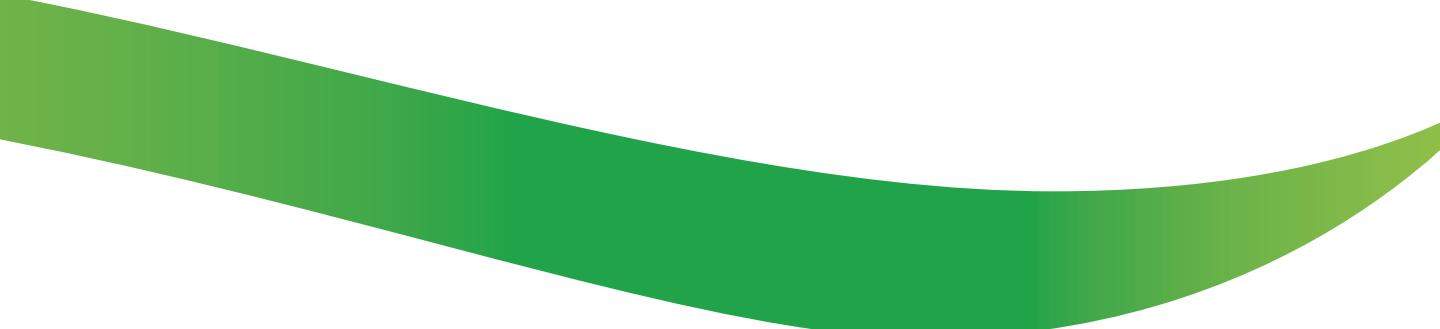

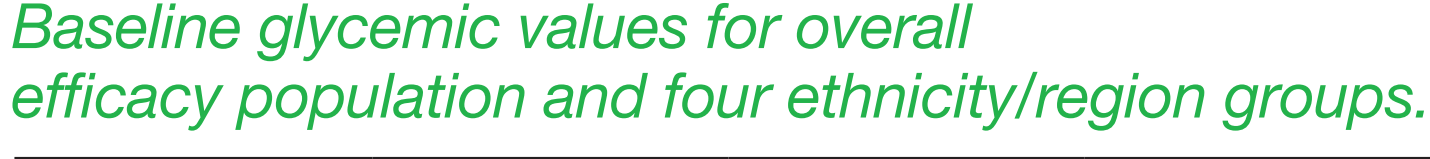

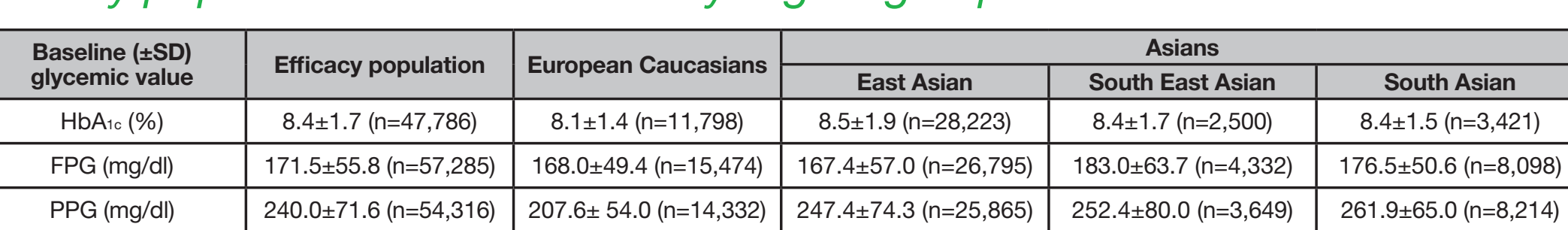

Changes in glycemic parameters at follow up: $\mathrm{HbA}_{1}$ Mos
to $7.3 \pm 1.3 \%$ at both the 3 -month visit $(n=34,571)$ and the last visit $(n=41,247)$
- Absolute reductions in patients with baseline and post-treatment $H b A_{10}$ data were $-1.12 \pm 1.31 \%$ at 3 -month visit $(n=32,692)$ and $-1.09 \pm 1.31 \%$ at the last visit
$(n=38,843)(p<0.0001$ for both $)$

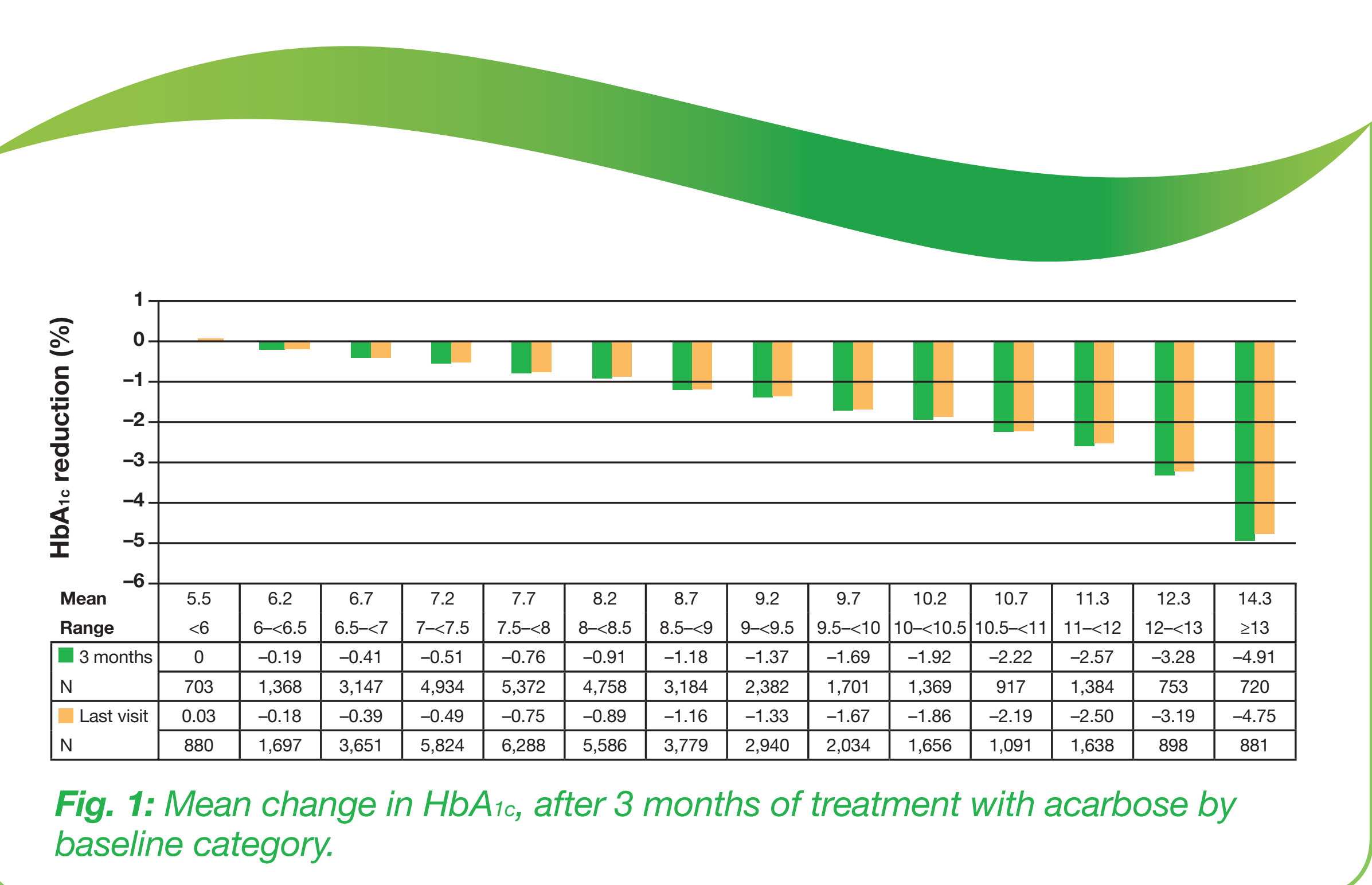

A descriptive plot of change in $H b A_{i c}$ versus baseline $H b A_{1 c}$ showed similar results

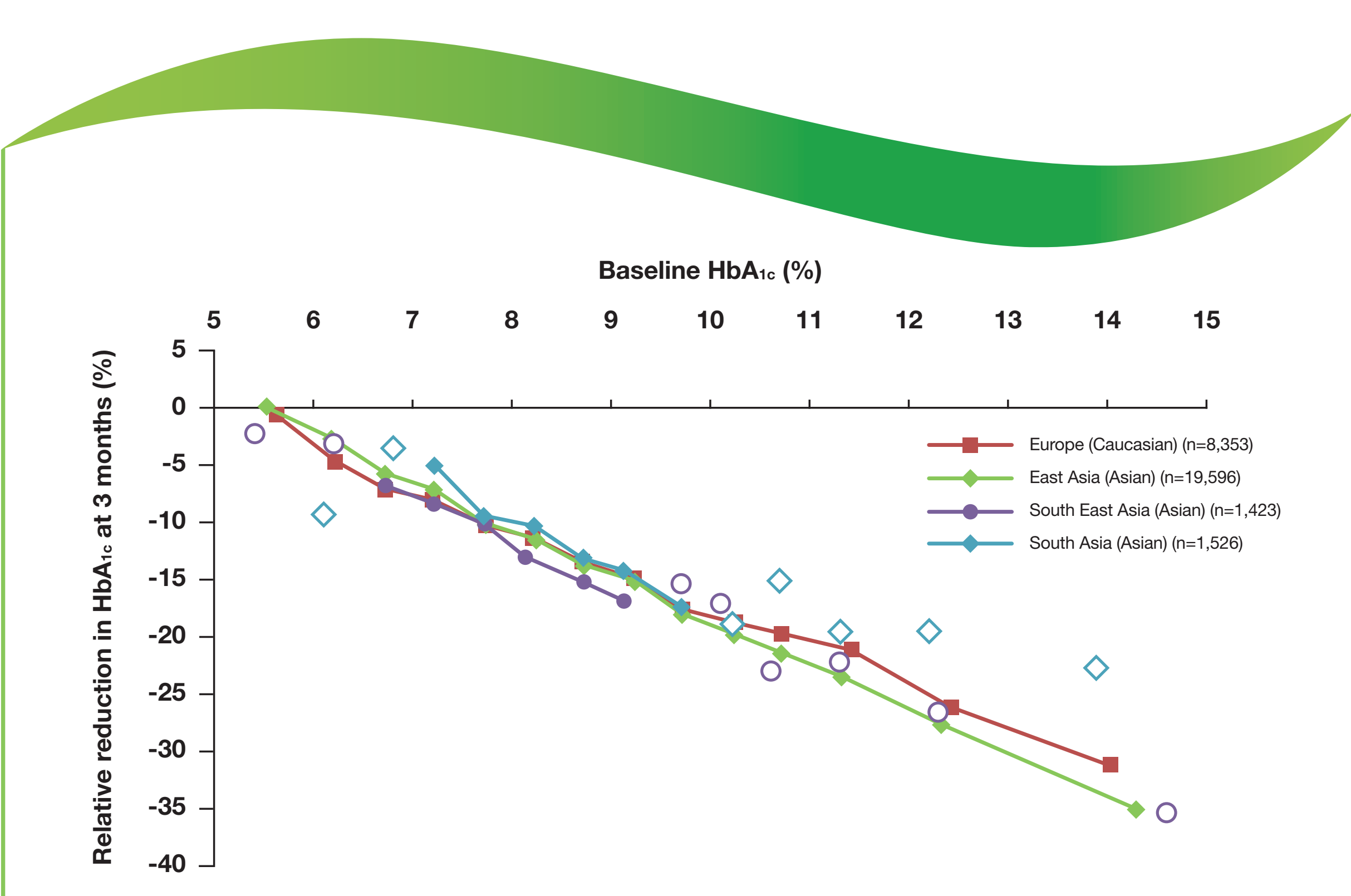

Fig. 2: Mean relative reduction vs mean baseline HbAtc by ethnicity/region
rour and baseline HbAtc category. Open symbols indicate $n<70$; baseline group and baseline HbAtc category. Open symbols indicate $n<70$; baseline
categories are as in Fig. 1 .

Regression lines for $\mathrm{HbAA}_{\mathrm{Ac}}$ change at 3 months versus baseline $\mathrm{Hb} \mathrm{A}_{1 \mathrm{c}}$ in each
ethnicity/region group indicated some differences in the observed efficacy of acarbose between ethnicity/region groups Relative reductions in $\mathrm{HbA} \mathrm{A}_{1 \mathrm{c}}$ ranged from $10.95 \%$ for South Asians to $12.98 \%$ for
South East Asians for mean baseline HbA1c $8.4 \%$ ( $p<0.0001)$ (Fig. 3$)$

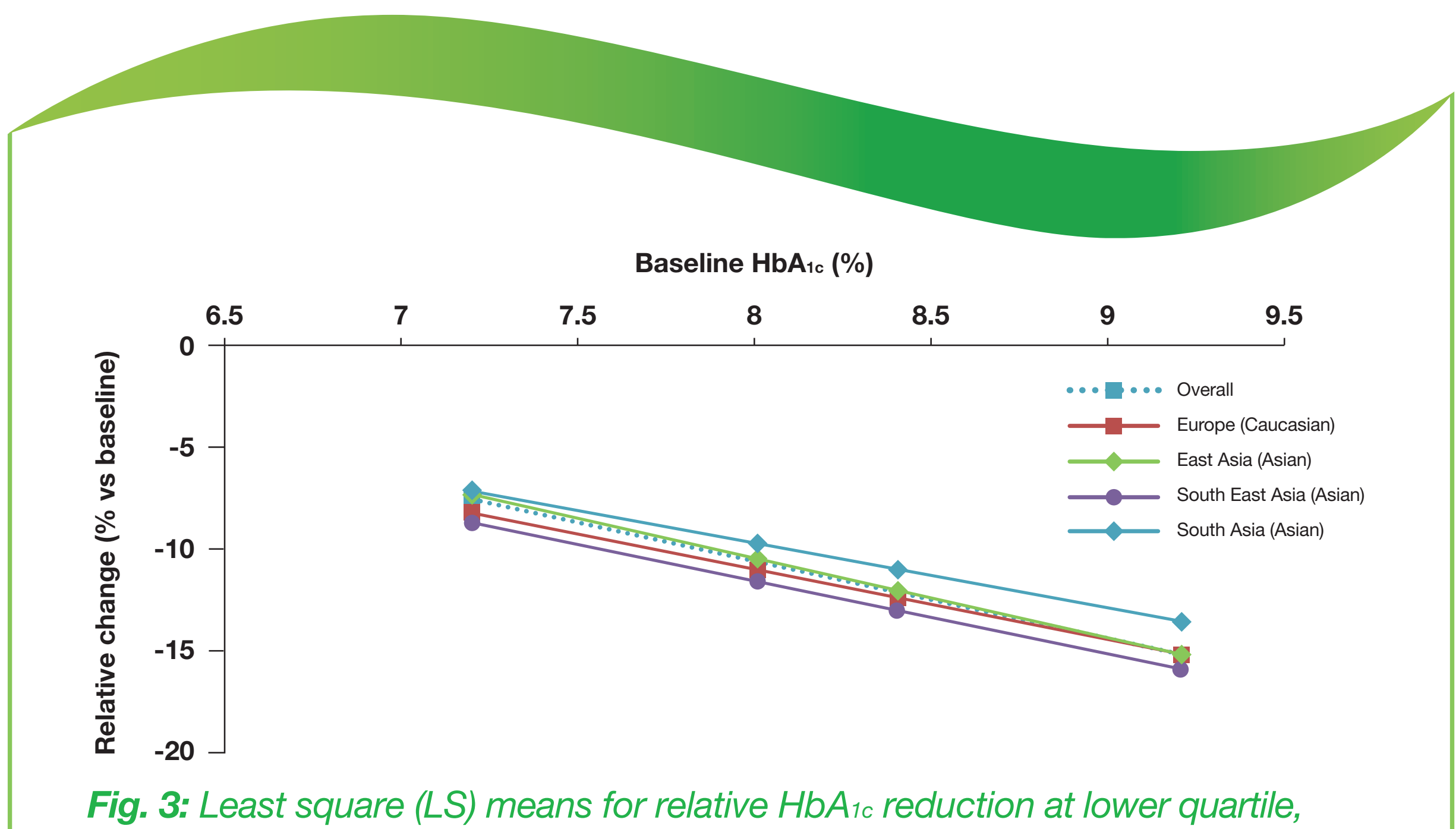

Fig. 3: Least square (LS) means for relative HbAtc reduction at lower quartile,

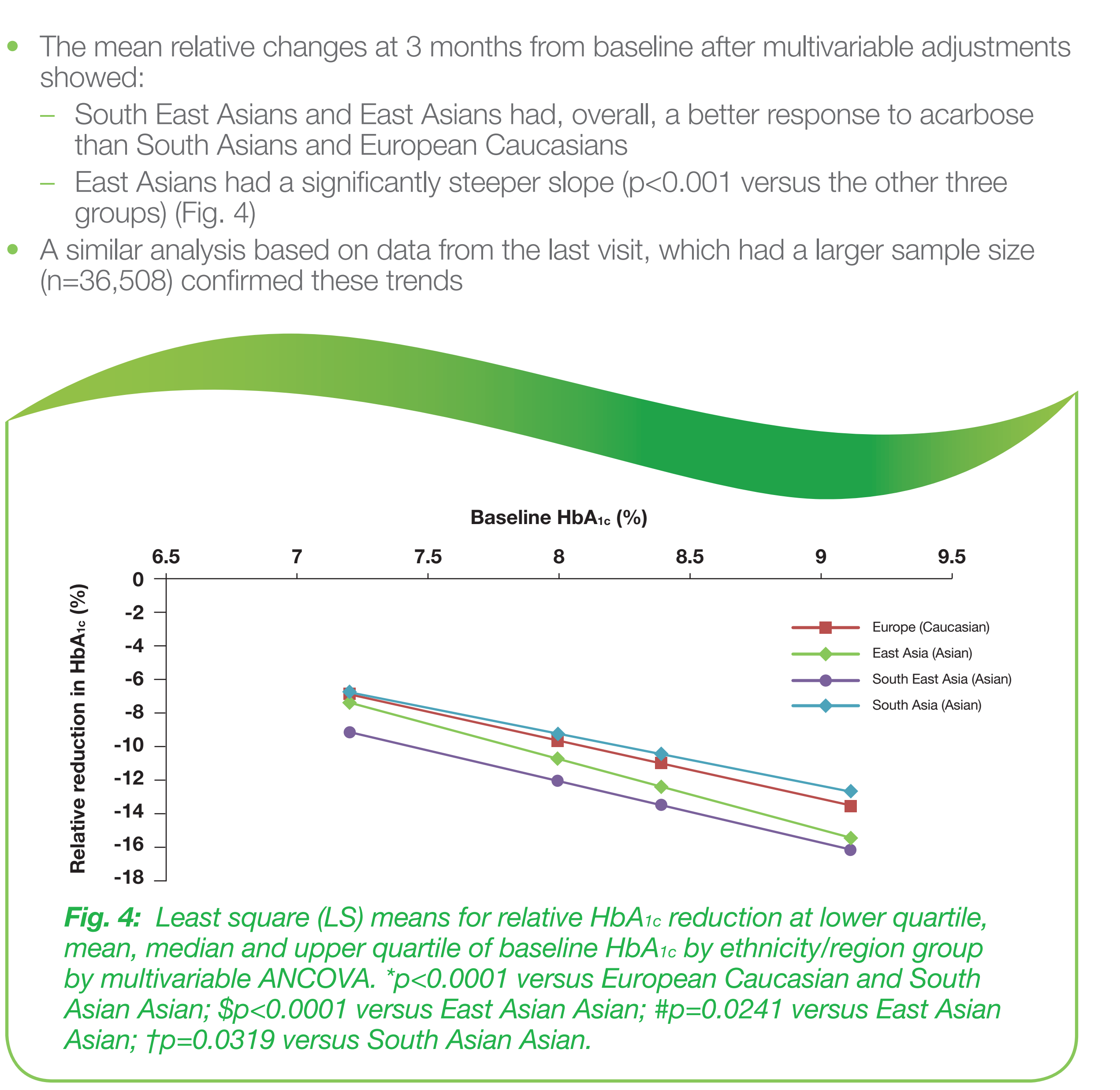

FPG

45,102 patients had non-missing baseline and 3-month data for FPG At the 3-month visit, FPG had decreased by $37.59 \pm 47.26 \mathrm{mg} / \mathrm{dl}$ from $170.2 \mathrm{mg} / \mathrm{dll}$ at Reductions in FPG were larger in patients with higher baseline FPG (Fig. 5)

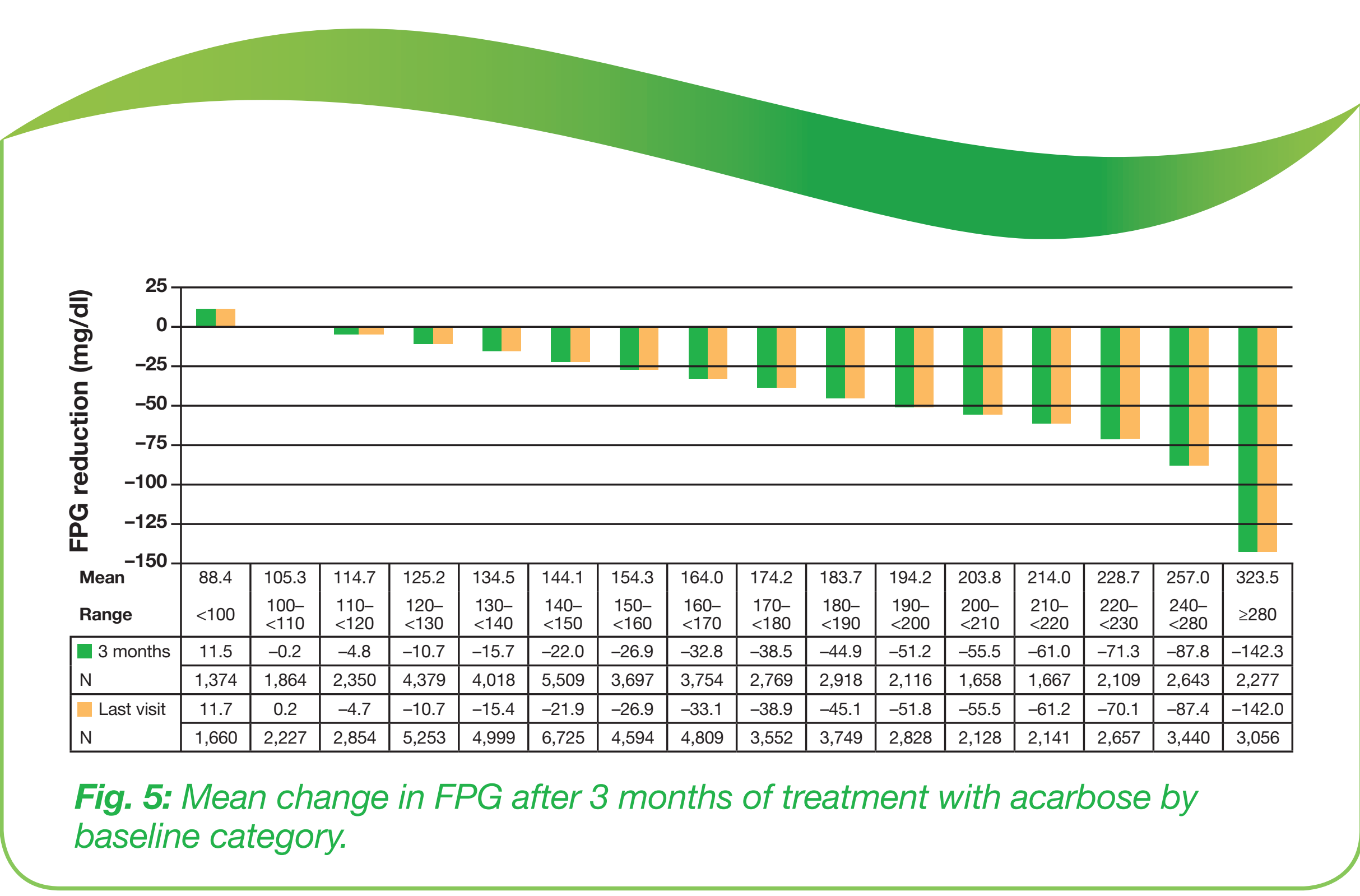

PPG

43,290 patients had non-missing baseline and 3-month data for PPG At the 3-month visit, PPG had decreased by $70.00 \pm 65.30 \mathrm{mg} / \mathrm{dl}$ from $238.2 \mathrm{mg} / \mathrm{dll}$ at Reductions in PPG were larger in patients with higher baseline PPG (Fig. 6

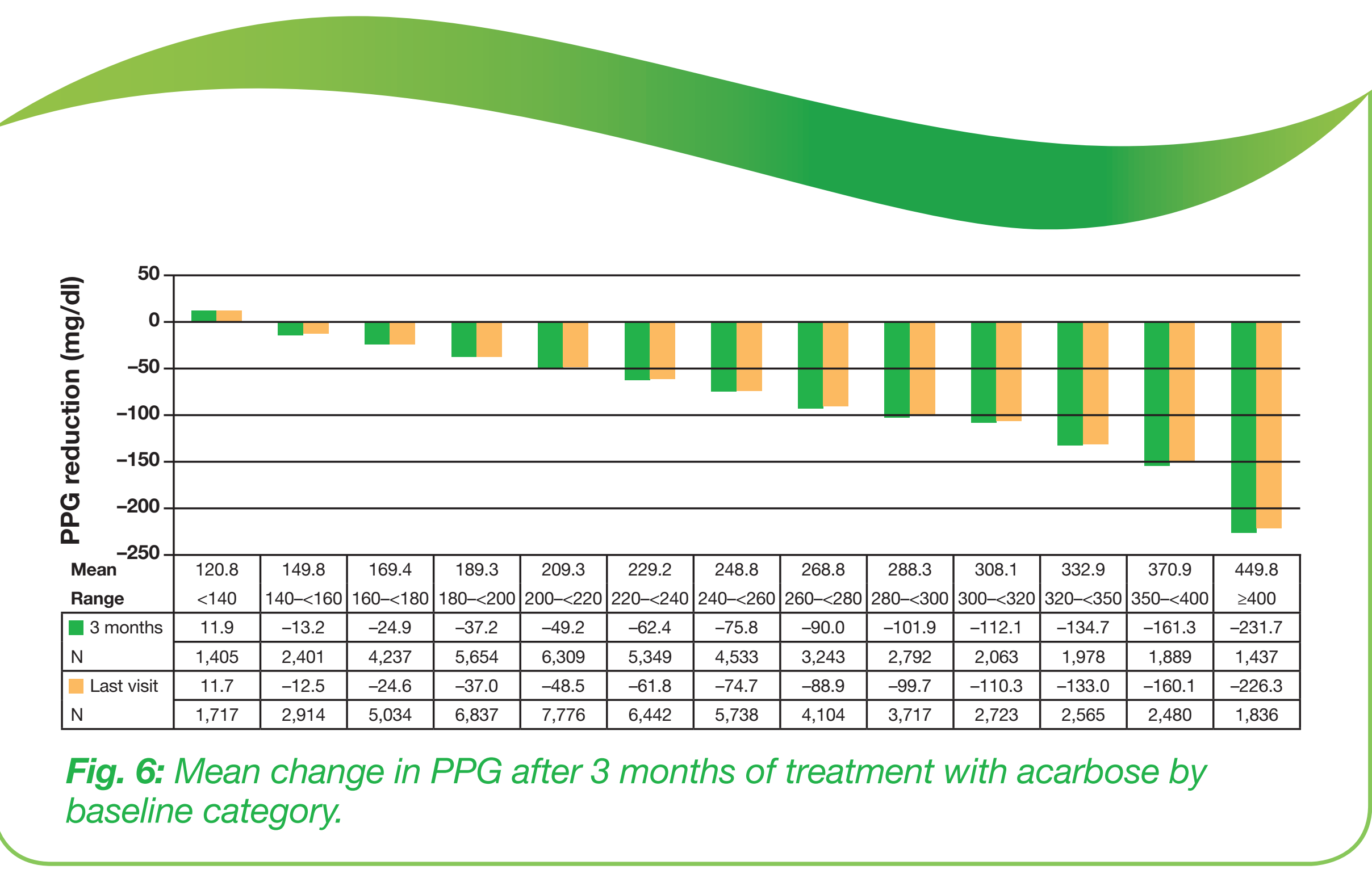

Safety

Acarbose was well tolerated with a low adverse event rate $(3.70 \%)$
Patients experienced very few episodes of hypoglycemia $(0.03 \%)$

The most frequently reported adverse events, as expected, were gastrointestinal (G) Study limitations

Our analysis includes individual patient data, and we were able to adjust for
confounding factors in individual patients, including baseline HbA $A_{1 c}$, which is well
known to affect the effect size with acarboses ${ }^{3,32}$. This is the first study to analyze differences in outcomes in clinical practice rather
than data from clinical trials, and reflects the change in $\mathrm{Hb} \mathrm{A}_{\mathrm{c}}$ from baseline that than data from clinical trials, and reflects the change in $\mathrm{Hb} \mathrm{A}_{10}$ from baseline that
physicians and patients can expect to see in real life Due to the nature of NIS, there was no control population (placebo group) for As a wide time span existed between the first and the last NIS and as different case Discussion/conclusion

This analysis of a large database of pooled data from real-life practice in diverse
patients from all over the world show that acarbose is effective across all ethnicities and regions examined, although the effects are more pronounced in some
populations, such as South East Asians and East Asians The relative reduction in $H b A_{1}$ w with acarbose is more pronounced in patients with
higher baseline $H b A_{1}$ values; this trend is more prominent in East Asians than other ethnicity/region groups South East Asians and East Asians have slightly but significantly better responses
to acarbose than South Asians and Caucasians from Europe after adjustment of Overall, acarbose has good efficacy regardless of ethnicity and region

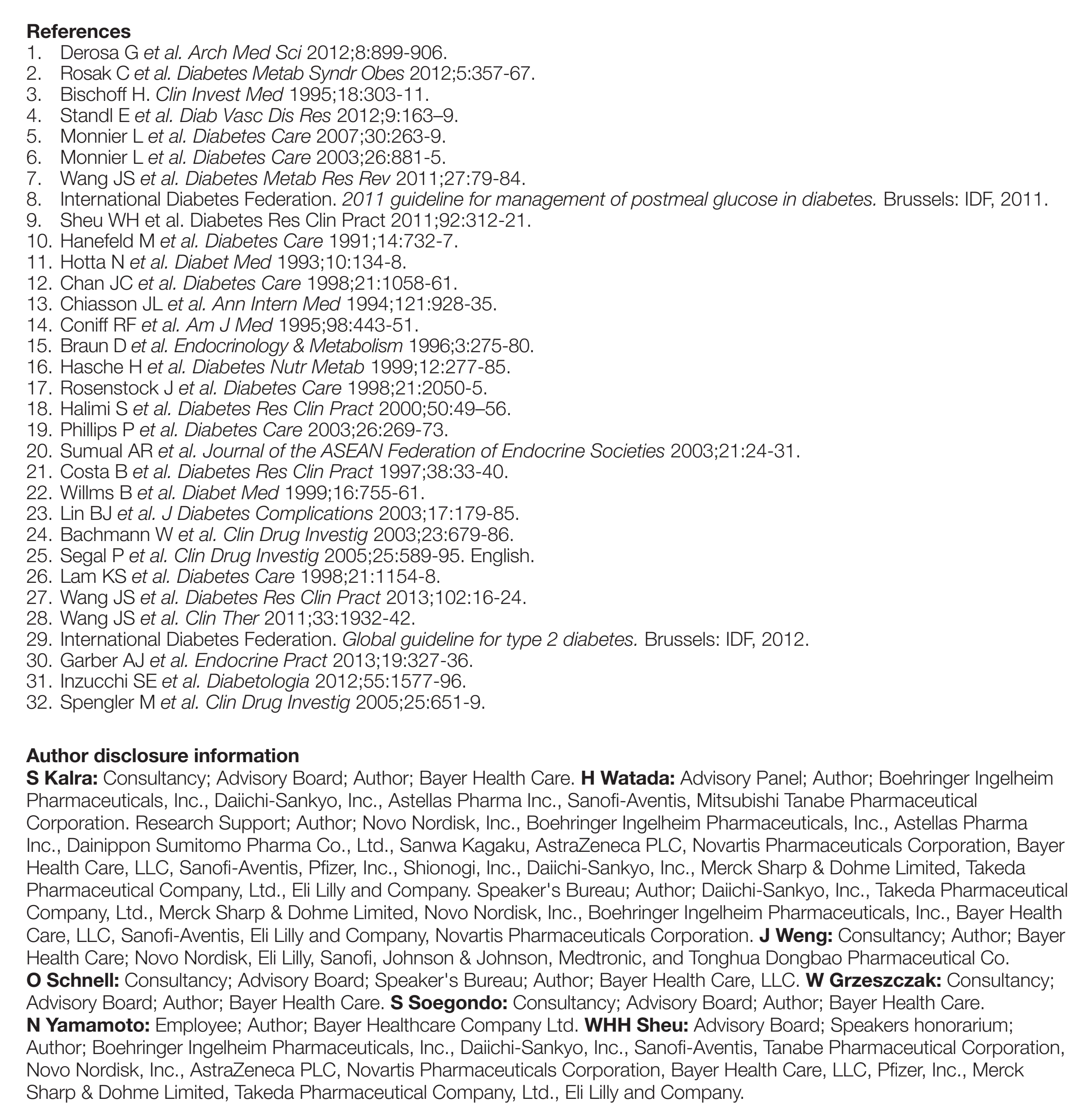

